\title{
Mobile augmented reality hand wash (MARHw): mobile application to guide community to ameliorate handwashing effectiveness to oppose Covid-19 disease
}

\begin{abstract}
The global concern about a new pandemic associated with the insufficiency of vaccines has necessitated basic practices of handwashing to get prevention in Covid-19 disease in modern health issues. At this point, due to that, the researchers develop a simple mobile application engaging augmented reality to educate the urban and rural community on a notable effect of basic infection prevention practices peculiarly on hand wash guidance to prevent Covid19infection. A quality of a hand wash could be attained by washing hands in conformity with health standard guidelines. In this study, we analyze a sample size of 83 participants $(n=83)$, there were categorized by age, young adult (ages 15 to $35, n=28$ ), middle-age (36 to $55, \mathrm{n}=28$ ) and older adults (older than 56 years, $\mathrm{n}=27$ ) through three main formulated hypotheses namely as a step, movement, and duration and being analyzed by SPSS version 25by using Anova. The differences in demographics andgender are not being compared amongst the group. The final result underlying a statistical significance draws two significantfactors for step and movement and there was no significant effect at the time factor of utilizing this mobile application. Hence,the final result could mitigate the Malaysian government sector peculiarly to the health department to alleviate the load of educating a community about a proper hand wash that would ease theprocess.
\end{abstract}

Keyword: Hand wash; Augmented reality and education 